\title{
THE CARDINAL POINTS IN CORRECTED AMETROPIA*
}

BY

\author{
Joseph I. Pascal \\ New York
}

IN hypermetropia the clear image of a distant object falls behind the retina; in myopia the clear image of a distant object falls in front of the retina. The correcting lens, convex or concave, does not alter the position of the retina, but alters the position of the clear image, placing it on the retina. The clear image formed by the optical system of the eye is best known as the optical image. Moving the optical image from behind, or in front of, the retina on to the retina is accomplished primarily by a movement of the cardinal points of the eye.

If the correcting plus lens is placed so that its second principal. point is at the anterior principal focus of the eye, the combined

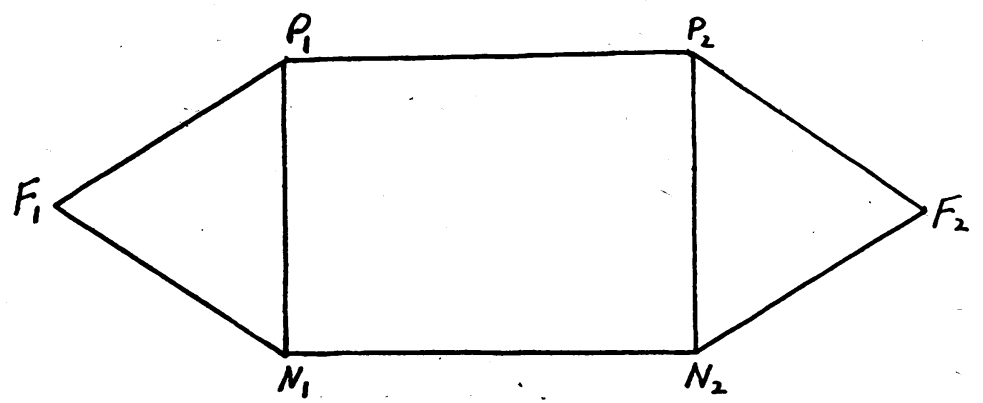

FIG. 1.-The cardinal points of the uncorrected eye.

power of eye and lens is the same as that of the eye alone. Similarly, if the correcting minus lens is placed so that its second principal point is at the anterior principal focus of the eye, the combined power of eye and lens is the same as that of the eye alone. The significant optical changes which these lenses produce is a shifting of the cardinal points of the eye. These shifts can be diagrammatically shown and remembered by a horizontally placed Benzene ring.

Let $\mathrm{F}_{1}, \mathrm{~F}_{2}, \mathrm{P}_{1}, \mathrm{P}_{2}, \mathrm{~N}_{1}, \mathrm{~N}_{2}$ (Fig. 1) represent the cardinal points of the uncorrected eye. These points are the two principal focal points, the two principal points, and the two nodal points. 
Distances represented by opposite parallel lines are equal : thus $\mathrm{P}_{1} \mathrm{~F}_{1}$ (the first principal focal length) equals $\mathrm{N}_{2} \mathrm{~F}_{2} ; \mathrm{P}_{2} \mathrm{~F}_{2}$ (the second principal focal length) equals $\mathrm{N}_{1} \mathrm{~F}_{1} ; \mathrm{P}_{1} \mathrm{P}_{2}$ (the distance between the principal points) equals $\mathrm{N}_{1} \mathrm{~N}_{2}$ (the distance between the nodal points); $P_{1} N_{1}$ equals $P_{2} N_{2}$ and represents the " equivalent" radius of the system, that is the radius of the single refracting surface which can replace the system.

In Fig. 2, let $\mathrm{F}_{1}^{\mathrm{c}}, \mathrm{F}_{2}^{\mathrm{c}}, \quad \mathrm{P}_{1}^{\mathrm{c}}, \quad \mathrm{P}_{2}^{\mathrm{c}}, \quad \mathrm{N}_{1}^{\mathrm{c}}$, and $\mathrm{N}^{\mathrm{c}}{ }_{2}$ represent the cardinal points after correction, i.e., of the combined system of lens and eve. If a plus lens is used for correction in

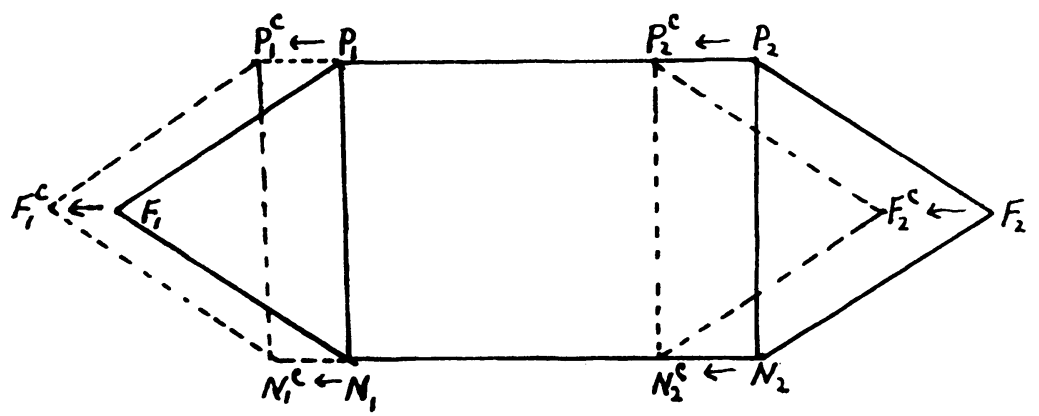

FIG. 2.-Pascal's schema for the movements of the cardinal points in corrected hypermetrofia.

the position previously mentioned, the position of the combined cardinal points with reference to the cardinal points of the eye alone is indicated in the figure.

All the cardinal points of the uncorrected eye move forwards (think of the cornea being to the left and the retina to the right). The direction of movement is indicated by little arrows. $\mathrm{P}_{1}, \mathrm{~N}_{1}$, and $\mathrm{F}_{1}$ all move the same amount. $\mathrm{P}_{1}^{\mathrm{c}} \mathrm{F}^{\mathrm{c}}{ }_{1}$ is parallel to $\mathrm{P}_{1} \mathrm{~F}_{1}$. So is $\mathrm{N}_{1}^{\mathrm{c}} \mathrm{F}^{\mathrm{c}}{ }_{1}$ parallel to $\mathrm{N}_{1} \mathrm{~F}_{1}$. The extent of this movement depends partly on the thickness of the correcting lens and is equal to the distance between the principal points of the lens. $\mathrm{P}_{2}, \mathrm{~N}_{2}$, and $\mathrm{F}_{2}$ also move an equal amount, ordinarily a greater distance than the movement of the first set of cardinal points. $\mathrm{P}^{\mathrm{c}}{ }_{2}$ $\mathrm{F}_{2}{ }_{2}$ and $\mathrm{N}_{2}{ }_{2} \mathrm{~F}^{\mathrm{c}}{ }_{2}$ are parallel to $\mathrm{P}_{2} \mathrm{~F}_{2}$ and $\mathrm{N}_{2} \mathrm{~F}_{2}$. Parallelism of the focal length lines shows that the focal lengths of the combined system are equal to the corresponding focal lengths of the original system. The distance between the new principal points and the corresponding nodal points is the same as before, i.e., $\mathrm{P}_{1}^{\mathrm{c}} \mathrm{N}_{1}^{\mathrm{c}}=$ $\mathrm{P}_{1} \mathrm{~N}_{1}=\mathrm{P}_{2}{ }_{2} \mathrm{~N}_{2}{ }_{2}=\mathrm{P}_{2} \mathrm{~N}_{2}$. This is to be expected as the combined optical system has the same power as before, and the "equivalent" radius of the combined system must be the same as the equivalent radius of the original system. 
The principal points are closer together as if the combined system were a " thinner" optical system. The nodal points are also closer together showing less displacement of the secondary axes. The second nodal point has moved forwards resulting in a larger retinal image as is the usual finding in corrected hypermetropia.

Fig. 3 shows what happens in a myopic eye with a minus lens correction. The "first" cardinal points, $\mathrm{P}_{1}, \mathrm{~N}_{1}$, and $\mathrm{F}_{1}$, move forwards; the " second" cardinal points, $\mathrm{P}_{2}, \mathrm{~N}_{2}$, and $\mathrm{F}_{2}$, move backwards. The movement of the " second " points is greater than

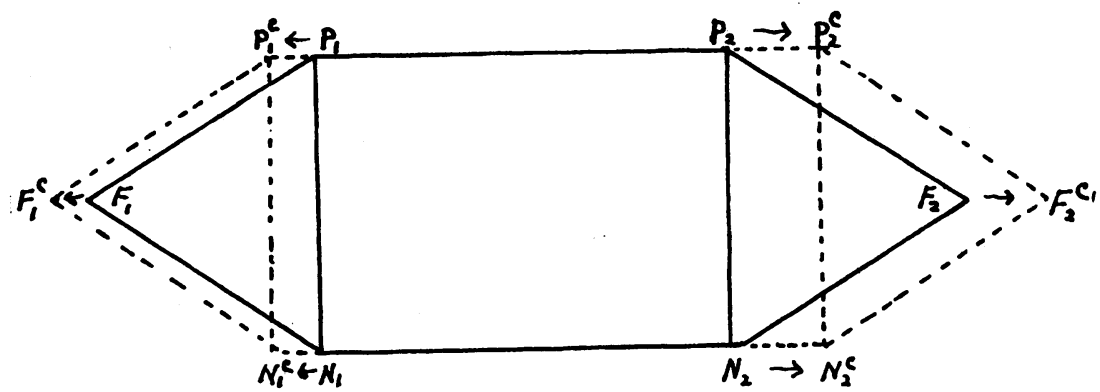

FIG. 3.-Pascal's schema for the movement of the cardinal points in corrected myopia.

the movement of the "first" points, the latter being equal to the distance between the principal points of the concave correcting lens. Here too parallelism of the lines shows that the focal lengths of the combined system are equal to the corresponding focal lengths of the original system. So is the " equivalent " radius of the combined system equal to the "equivalent" radius of the original system. The principal points are further apart as if the combined system is a " thicker" optical system.

The nodal points are also further apart, showing greater displacement of the secondary axes. The second nodal point has moved backwards (towards the retina), producing a smaller retinal image than the "basic" retinal image before correction. By the " basic " image is meant the image formed by the points of intersection of the secondary axes with the retina. The diffusion circles in the uncorrected image make the original image even larger. The resultant smaller retinal image is the usual finding in corrected myopia.

The Benzene ring can be used in the same fashion to visualize the movement of the cardinal points when the correcting lens is placed in a position different from the one considered in the present article. 


\section{Summary}

A graphic method is presented to show visually the movement of the cardinal points of an eye occasioned by the application of correcting lenses placed in the plane of the anterior principal focus of the eye. The resultant changes in the "thickness" of the optical system, the displacement of the secondary axes, the increase or decrease in the size of the retinal image, etc., can be read off directly from the schema and more easily remembered and understood.

\section{OBITUARY}

\section{MARCUS FINE}

WE regret to announce the recent death of Marcus Fine in Johannesburg as a result of a motor accident.

He qualified from the London Hospital in 1925 and ultimately became assistant, and then senior, House Surgeon to the Royal Westminster Ophthalmic Hospital in 1927-28. He was the last House Surgeon to the hospital on its old site adjacent to Charing Cross. He obtained the D.O.M.S. in 1927, and on returning to South Africa built up a flourishing ophthalmic practice in Johannesburg, where he was later appointed Assistant Ophthalmic Surgeon to the General Hospital. When he re-visited London in 1949, he took considerable interest in the operation and orthoptic work at his old hospital.

M. L. H. 